\title{
Childhood maltreatment and suicidal ideation in Chinese children and adolescents: The mediation of resilience
}

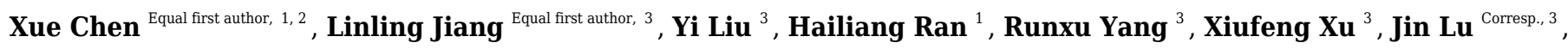 \\ Yuanyuan Xiao ${ }^{\text {Corresp. } 1}$ \\ ${ }^{1}$ School of Public Health, Kunming Medical University, Kunming, China \\ 2 The Second Affiliated Hospital, Kunming Medical University, Kunming, China \\ 3 The First Affiliated Hospital, Kunming Medical University, Kunming, China \\ Corresponding Authors: Jin Lu, Yuanyuan Xiao \\ Email address: Jinlu2000@163.com, 33225647@qq.com
}

Background. Childhood maltreatment could increase the risk of suicidal ideation (SI) in adolescents. However, the mediation of resilience in this association remains unclear. Method. A population-based cross-sectional study has been done among 3,146 Chinese adolescents. We collected relevant information from the study participants by using selfadministered questionnaire. Chinese version of the Childhood Trauma Questionnaire (CTQ), the Resilience Scale for Chinese Adolescents (RSCA), and the Beck Scale for Suicide Ideation (BSSI) were used to measure childhood maltreatment, resilience, and SI, respectively. Univariate and multivariate binary Logistic regression models were employed to estimate crude and adjusted associations between childhood maltreatment, resilience, and SI. Path analysis has subsequently been performed to measure the mediation of resilience in this association. Results. Multivariate Logistic regression models revealed that compared to non-abused counterparts, adolescents who had ever experienced any type of childhood maltreatment was 1.74 times likely to report SI. Among the specific types of childhood maltreatment, emotional abuse showed the strongest association with SI (adjusted OR=3.01, 95\% Cl: 2.37-3.82). Path model suggested that over one-third $(39.8 \%)$ of the total association between childhood maltreatment and SI was mediated via resilience. Emotion regulation and interpersonal assistance were the most prominent mediators among all dimensions of resilience. Conclusions. Resilience played as a significant mediator in the association between childhood maltreatment and SI. Resilienceoriented intervention measures could be considered for suicidal risk prevention among abused Chinese adolescents. 


\section{Childhood maltreatment and suicidal ideation in Chinese}

\section{2 children and adolescents: The mediation of resilience}

4 Xue Chen ${ }^{\text {a, b, } \# \quad \text { Linling Jiang }}{ }^{\mathrm{c}, \#}$ Yi Liu $^{\mathrm{c}} \quad$ Hailiang Ran ${ }^{\mathrm{a}}$

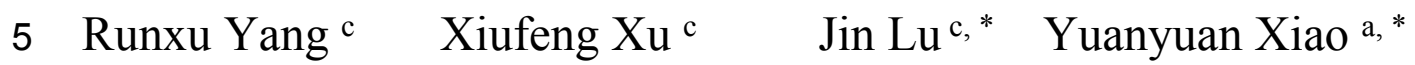

$7 \quad{ }^{a}$ School of Public Health, Kunming Medical University, Kunming, Yunnan, China

$8{ }^{\mathrm{b}}$ The Second Affiliated Hospital, Kunming Medical University, Kunming, Yunnan, China

$9{ }^{\mathrm{c}}$ The First Affiliated Hospital, Kunming Medical University, Kunming, Yunnan, China

10

11 \# These authors contributed equally as joint first authors.

14 * Correspondence to:

Dr. Jin Lu, Department of Psychiatry, The First Affiliated Hospital, Kunming Medical University, Kunming, Yunnan, China. E-mail: Jinlu2000@163.com;

Dr. Yuanyuan Xiao, Department of Epidemiology and Health Statistics, School of Public Health, Kunming Medical University, Kunming, Yunnan, China. 650500. E-mail: 33225647@qq.com 


\section{Abstract}

24 Background. Childhood maltreatment could increase the risk of suicidal ideation (SI) in 25 adolescents. However, the mediation of resilience in this association remains unclear.

26 Method. A population-based cross-sectional study has been done among 3,146 Chinese 27 adolescents. We collected relevant information from the study participants by using self28 administered questionnaire. Chinese version of the Childhood Trauma Questionnaire (CTQ), the 29 Resilience Scale for Chinese Adolescents (RSCA), and the Beck Scale for Suicide Ideation 30 (BSSI) were used to measure childhood maltreatment, resilience, and SI, respectively. Univariate 31 and multivariate binary Logistic regression models were employed to estimate crude and 32 adjusted associations between childhood maltreatment, resilience, and SI. Path analysis has 33 subsequently been performed to measure the mediation of resilience in this association.

34 Results. Multivariate Logistic regression models revealed that compared to non-abused counterparts, adolescents who had ever experienced any type of childhood maltreatment was 1.74 times likely to report SI. Among the specific types of childhood maltreatment, emotional 37 abuse showed the strongest association with SI (adjusted OR=3.01, 95\% CI: 2.37-3.82). Path model suggested that over one-third $(39.8 \%)$ of the total association between childhood maltreatment and SI was mediated via resilience. Emotion regulation and interpersonal assistance were the most prominent mediators among all dimensions of resilience.

41 Conclusions. Resilience played as a significant mediator in the association between childhood 42 maltreatment and SI. Resilience-oriented intervention measures could be considered for 43 suicidal risk prevention among abused Chinese adolescents.

44 Keywords. Childhood Maltreatment; Suicidal Ideation; Resilience; Adolescents 


\section{Introduction}

47 Suicide is one leading cause of death among adolescents worldwide (WHO, 2018). The 48 suicidality model emphasizes the continuous developmental stages in sequence: suicidal ideation 49 (SI), suicide plan, suicide attempt, and finally, completed suicide (Sveticic \& De Leo, 2012). 50 Based on this theory, suicidal risk evolves from low to high with the progression along this path 51 (Szanto et al., 2003). From this point of view, SI can be seen as an imminent precursor of the 52 subsequent suicide action, thus identifying suicide ideators is critical for effective suicide 53 intervention.

54 SI can be influenced by multiple indicators among adolescents, like age, gender, mental 55 disorders, educational levels, social support, interpersonal difficulties, and family conflict 56 (Ahorsu et al., 2020; Salama et al., 2020; Xiao et al., 2019). In addition, a large body of studies 57 suggested that childhood maltreatment is a salient risk factor for SI (Falgares et al., 2018; 58 Stickley et al., 2020). Childhood maltreatment is defined as the abuse or neglect of children 59 under the age of $16(C D C, 2017)$. It has been corroborated that childhood maltreatment can 60 significantly increase future risk of affective disorders, such as depression, anxiety, borderline 61 personality disorder, behavioral problems, or even suicide (Rehan et al., 2017; Rafiq, 62 Campodonico, \& Varese, 2018; Fry, McCoy, \& Swales, 2012).

63 Childhood maltreatment is also a concern in China. Previous studies have revealed that $36.6 \%$ 64 of the Chinese population reported physical child abuse experience ( $\mathrm{Ji} \&$ Finkelhor, 2015), the 65 prevalence of childhood sexual abuse was $24.8 \%$ and $17.6 \%$ for female and male college 66 students (Chen, Han, Lian, \& Dunne, 2010). Although the positive association between 67 childhood abuse and SI has been well established, mechanism underneath this association, 68 specifically, possible mediators in this association, remains unclear. 
69 In the field of psychology, resilience has been defined as the ability to thrive in hardships or to 70 recover from negative events (de Terte \& Stephens, 2014). Adolescent resilience is a multi-layer 71 concept, which contains characteristics from individual level (such as goal concentration, 72 emotion regulation, positive perception), family level (such as family support), and social 73 environment level (such as interpersonal assistance) (Olsson et al., 2003). Prior studies suggested 74 that children with childhood maltreatment experience had significantly lower psychological 75 resilience (Dubowitz et al., 2016), meanwhile, it has been corroborated that psychological 76 resilience level was significantly associated with the occurrence of SI (Cong et al., 2019). 77 Therefore, it is logical to suspect that resilience may play as a mediator in the association 78 between childhood maltreatment and SI. However, to our best knowledge, existing literature did 79 not thoroughly investigate this hypothesis among Chinese adolescents.

80 Aimed to address this issue, in this cross-sectional study, we intended to estimate the possible 81 mediation of resilience in the association between childhood maltreatment and SI in a large 82 group of Chinese adolescents. We put forward two hypotheses: 1) Different types of childhood 83 maltreatment experiences are significantly but discordantly associated with increased risk of SI;

84 2) Resilience exerts prominent mediation in the association between childhood maltreatment and 85 SI.

\section{Materials \& Methods}

88

89

90

\section{Participants}

A cross-sectional survey was conducted in Lincang, western China Yunnan province from December 1 to December 13, 2019. Participants were selected using a multistage simple random cluster sampling method. In stage one, we randomly chose Linxiang district from a total of 8 
92 districts within Lingcang; in stage two, 5 primary schools, 5 junior middle schools, and 4 senior

93 middle schools were selected randomly; at last, 3 to 4 classes were randomly selected in each

94 chosen school based on the required sample size. All eligible students in these classes were

95 preliminarily included. The process for sampling and study participants selection was shown in

96 Figure 1. The questionnaires were self-administered by respondents. When filling in the

97 questionnaires, any confusion of the respondents can be consulted immediately at sites. To avoid

98 any potential information lost, each completed questionnaire was also carefully reviewed

99 immediately by pre-trained quality control personnel.

100 Adolescents aged above 10 and below 18 were eligible study subjects, the rationale for a lower

101 limit of 10 was based on the finding that a child cannot well understand the concept of suicide

102 until the age of 10 (Mishara BL, 1999). Children and adolescents were further screened by using

103 the following exclusion criteria: (1) Illiterate; (2) Physically ill, cannot finish the survey; (3)

104 Auditory dysfunction or language disorder; (4) Unconscious or delirious, cannot clearly express

105 oneself. The presence of criteria (2)-(4) had been carefully evaluated by professional clinicians

106 deployed at the survey sites. Prior to the survey, written consents were obtained from both the

107 participants and their legal guardians. The study protocol was reviewed and approved by the

108 Third People's Hospital of Lincang Ethics Committee (Approval number: 2019-01).

109 2. Measurements

110 2.1. General characteristics. General characteristics of the participants, mainly demographics

111 (gender, age, ethnicity, residence, grade, study style) and socioeconomic status (father's age, 112 mother's age, father's education level, mother's education level, family income, marital status of 113 the parents), were collected by using a self-developed questionnaire. 
114 2.2. Childhood maltreatment. Chinese version of the Childhood Trauma Questionnaire (CTQ), a

115 self-report scale of 28 items, was used to assess childhood physical abuse (PA), emotional abuse

116 (EA), sexual abuse (SA), physical neglect (PN), and emotional neglect (EN). A 5-point scale

117 rated answers are used for each question: $1=$ never, $2=$ rarely, $3=$ sometimes, $4=$ often, and $5=$ very

118 often. The total score for each type of maltreatment ranges between 5 and 25 . The following

119 recommended cut-offs were used to dichotomize study subjects: 8 for PA, 9 for EA, 6 for SA, 8

120 for PN, 10 for EN (Bernstein et al., 1994). The Chinese version of CTQ showed acceptable

121 reliability (Zhao, Zhang, Li, \& Zhou, 2005).

122 2.3. SI. We used Chinese version of the Beck Scale for Suicide Ideation (BSSI) to assess lifetime 123 SI of the participants (Beck, Steer, \& Ranieri, 1988). BSSI is one of the few suicide assessment 124 instruments that possess predictive validity for completed suicide (Zhang \& Brown, 2007). 125 Participants with one of the following two conditions were defined as suicide ideator: 1) 126 Answered "Weak" or "Moderate to strong" to the question "Desire to make active suicide 127 attempt"; 2) Answered "Would leave life/death to chance" or "Would avoid steps necessary to 128 save or maintain life" to the question "Passive suicidal desire" (Xiao et al., 2019).

129 2.4. Resilience. The 27-item Resilience Scale for Chinese Adolescents (RSCA) was used to 130 evaluate the 5 dimensions of resilience (goal concentration, emotion regulation, positive 131 perception, family support, and interpersonal assistance). RSCA was designed by Hu and Gan 132 (2008), and its reliability and validity have been corroborated in Chinese adolescents. The higher 133 combined RSCA score reflects a higher level of resilience. The Cronbach's $\alpha$ for RSCA based 134 on our analytical sample was 0.82 (Bootstrap 95\% CI: 0.80-0.83).

135 3. Statistical analysis

Peer) reviewing PDF | (2021:01:57531:2:0:NEW 2 Jun 2021) 
136 Descriptive statistics were used to describe and compare general characteristics. We used

137 univariate and multivariate binary Logistic regression models to explore the crude and adjusted

138 associations between childhood maltreatment, resilience, and SI. At first, univariate model was

139 used to screen for prominent demographic and socioeconomic covariates of SI at a lower

140 significance level of 0.10 . Prominent variables discerned by univariate models were further

141 included into the subsequent multivariate models. Childhood maltreatment and resilience were

142 analyzed separately in model 1 and 2 , then collectively in model 3 . Based on the results of

143 univariate and multivariate models, hypothetical path models were constructed to evaluate the

144 mediation of resilience in the association between childhood maltreatment and SI.

145 All analyses were performed by using the $\mathrm{R}$ software (Version 3.6.2). Possible inter-

146 correlation which caused by clustering sampling design was adjusted for by using $\mathrm{R}$ analytical

147 packages for survey data, such as "survey" and "lavaan. survey". The significance level was set

148 as $p<0.05$, two-tailed.

149

150 Results

151 General features of study participants

152 A total of 3,234 eligible adolescents were identified by using the inclusion and exclusion criteria,

15347 students cannot participate because they asked for sick leave, with a survey response rate of

$15498.5 \%$. Among the respondents, 3,146 provided valid and complete information, and the 155 effective response rate was $97.3 \%$. Characteristics of all included participants were displayed in

156 Table 1. A total of 1,091 adolescents reported SI, accounted for 34.7\% (95\% CI: 28.4\%-48.0\%).

157 Besides, a higher prevalence of SI was observed in girls. CTQ scores for all five types of 158 childhood maltreatment were significantly higher for suicidal ideators $(p<0.01)$. The mean of 
159 combined resilience score was 93.9 for all participants, and suicidal ideators showed lower

160 scores in all five dimensions of resilience $(p<0.01)$.

161 Associations between childhood maltreatment, resilience, and SI

162 Based on the cut-offs provided above for different dimensions of CTQ, 2,181 adolescents 163 reported at least one type of childhood maltreatment, accounting for $69.3 \%$ (95\% CI: $61.9 \%$ $16476.0 \%$ ). We used the median of RSCA (94) to dichotomize study participants as no commonly 165 used cut-off for RSCA has been recommended. Gender, age, grade, study style, ages of both 166 parents, education level of father, and marital status of the parents were significant covariates 167 included into the subsequent multivariate models. The fitting results of the final multivariate 168 model (Multivariate 3 in Table 2) indicated that: grade, study style, marital status of the parents 169 were significantly associated with SI, participants of higher grade, were day students, whose 170 parents were divorced, re-married, or widowed were observed increased risk of SI. Moreover, 171 after adjusted for prominent covariates, for adolescents who had ever experienced childhood 172 maltreatment, the odds of SI was 1.91 times (95\% CI: 1.51-2.41) compared to non-abused 173 adolescents; participants with a higher level of resilience (RSCA score $\geq 94$ ) were $80 \%$ less likely 174 (95\% CI: $74 \%-84 \%)$ to report SI than participants with a lower level of resilience (RSCA 175 score $<94)$.

176 Considering of the positive relationship between childhood maltreatment and SI, we further 177 investigated the associations between different types of child maltreatment and SI, and the results 178 were summarized in Figure 2: all types of maltreatment were associated with prominently 179 increased odds of SI, particularly, adolescents who had experienced emotional abuse showed the 180 highest SI risk (adjusted OR=3.01, 95\% CI: 2.37-3.82), followed by emotional neglect (adjusted $181 \mathrm{OR}=1.78,95 \% \mathrm{CI}: 1.35-2.36$ ), sexual abuse (adjusted OR=1.68, 95\% CI: 1.21-2.33), physical 
182 abuse (adjusted $\mathrm{OR}=1.53,95 \% \mathrm{CI}: 1.24-1.88$ ), and physical neglect (adjusted $\mathrm{OR}=1.34,95 \% \mathrm{CI}$ :

183 1.14-1.56).

184 Mediation of resilience in maltreatment-SI association

185 Based on the previous analytical results, a hypothetical path model was put forward to illustrate 186 the associations between childhood maltreatment, SI, and resilience. The path model justified 187 that $39.8 \%$ of the association was mediated via resilience. Then we fitted a series of path model 188 for 5 types of childhood maltreatment separately: after controlled for other related factors, EA, $189 \mathrm{EN}$, and PN were indirectly associated with SI through resilience, especially for EN and PN, as 190 their direct associations with SI became insignificant after adjusted for the mediation of 191 resilience (Figure 3).

192 We further discussed the mediation of resilience in the association between EA, EN, PN, and 193 SI by using its dimensions. Results showed that except for positive perception, the other four 194 dimensions of resilience were salient mediators. Emotion regulation showed the strongest 195 mediation for individuals who had exposed to EA, whereas for EN and PN, the strongest 196 mediator was interpersonal assistance (Figure 4).

197

198 Discussion

199 In this cross-sectional study of 3,146 Chinese children and adolescents, we found a strong 200 association between childhood maltreatment and SI, and among all types of childhood 201 maltreatment, emotional abuse showed the strongest association with SI. More importantly, as 202 hypothesized, resilience prominently mediated the association between childhood maltreatment 203 and SI: accounted for $39.8 \%$ of the total association. When dissecting resilience by using its 204 dimensions, emotion regulation and interpersonal assistance were the most prominent 
205 mediators. The major findings of our study can help understand the intimate relationship 206 between childhood maltreatment and SI, shed new light in the prevention of suicidal behaviors 207 which stem from the past child abuse experiences among Chinese children and adolescents.

208 In the current study, we found that SI was prevalent among Chinese children and adolescents, 209 as a lifetime prevalence of $34.7 \%$ has been found. This result was comparable to a previous study 210 (32\%) (Tan, Xia, \& Reece, 2018). Based on the analytical results, all types of childhood 211 maltreatment were significantly and positively associated with SI. The positive connection 212 between childhood maltreatment and SI is well supported by existing literature. First, childhood 213 maltreatment could disturb the hypothalamic-pituitary-adrenal axis. It has been found that adult 214 women with childhood abuse experience had higher plasma cortisol levels than non-abused 215 individuals, and elevated cortisol is an identified risk factor for future SI (Shalev et al., 2019). 216 Second, childhood maltreatment impairs cognitive function (Miller \& Esposito-Smythers, 2013), 217 and it has been repeatedly verified that cognitive impairment is directly associated with SI $(P u$, 218 Setoyama, \& Noda, 2017).

219 The results of path model revealed that over one-third of the association between childhood 220 maltreatment and SI was inversely mediated via resilience. Although no studies on exactly the 221 same topic can be compared with, it has been disclosed that resilience significantly mitigated 222 suicidal risk associated with childhood trauma (Poole, Dobson, \& Pusch, 2017; Meng et al., 223 2018). Two common perspectives currently exist regarding to the concept of resilience: the trait224 oriented perspective and the process-oriented perspective, and the latter is more widely accepted 225 by researchers, for it emphasizes that resilience is a process of, or the outcome of, or the capacity 226 for successful adaptation after setbacks. In other words, resilience can be modified. Fergus and 227 Zimmerman (2005) have proposed that internal "assets" (such as perceived ability, coping 
228 strategies, self-efficacy) and external "resources" (typically different types of social support) are 229 particularly critical in building up resilience of the adolescents.

230 Among all five types of childhood maltreatment, resilience played a significant mediation role 231 in their associations with SI only for EA, EN, and PN. Especially for EN and PN, as their direct 232 associations with SI were completely mediated by resilience. After careful literature review, we 233 have not identified any studies that attempted to discuss and compare the mediation of resilience 234 in associations between different types of childhood maltreatment and SI among adolescents. 235 However, a previous study found that, among all types of child abuse, ego-resiliency only 236 significantly mediated the relationships between EA, PN, EN and three psychological symptoms 237 (depression, self-harm behaviors, anxiety) (Hong, 2017). Emotional neglect in adolescents may 238 lead to shame, behavioral avoidance, and a dysfunctional attachment style, which then will 239 increase the risk of depression and anxiety, two recognized risk factors of SI (Lee et al., 2018).

240 Interpersonal assistance, suggested by our analytical results, was the strongest mediator in the 241 association between childhood neglect (either EN or PN) and SI among the 5 dimensions of 242 resilience measured in RSCA. For children and adolescents, the most prominent interpersonal 243 relationship involves friends at school (Eccles \& Roeser, 2011). Therefore, interpersonal 244 assistance that we measured in this study was largely peer support. In fact, friends and peers are 245 critical in the development of social relationships and interpersonal connectedness during the 246 stage when children and adolescents begin to form bonds outside of their families (Gorrese \& 247 Ruggieri, 2012). Thus, close friends and peers logically become the primary source of intimacy 248 and social support at this stage of life (Wilkinson, 2004). Peer support has been identified as a 249 protective factor against psychological problems, especially depression, among children and 250 adolescents (Sticeet al., 2011; Mizuta et al., 2017). Yearwood et al. (2019) have found that 
251 higher level of peer support could mitigate internalizing and externalizing psychopathological 252 symptoms associated with abuse and neglect experience in adolescents. Besides, by surveying 253 8,778 Chinese adolescents, Cui et al. (2010) observed that the lack of peer support was 254 significantly related to increased risk of SI. However, it is highly possible that the relationship 255 between interpersonal support and SI can be inverse, as our analysis was based on cross256 sectional data, and this possible inverse association can also be supported by previous 257 publications (Wiklander, Samuelsson, \& Asberg, 2003). Nevertheless, under any situation, the 258 importance of interpersonal assistance, especially peer support, should be recognized when 259 designing and implementing SI prevention strategies for adolescents who had experienced 260 childhood neglect.

261 Another important finding is that, for all dimensions of resilience, emotion regulation 262 presented the strongest mediation in the association between EA and SI. Besides, emotional 263 regulation also played a salient role in mediating the relationship between child neglect and SI. 264 All these findings suggest that improving emotion regulation ability can be another promising 265 way to help adolescents suffering from EA, EN and PN antagonize the risk of SI. Fortunately, 266 some effective intervention programs targeting at optimizing emotion regulation and emotional 267 competence among adolescents have already been implemented, such as Mastering emotions 268 technique (MEMT) (Patel, Nivethitha, \& Mooventhan, 2018), emotional schema therapy 269 (Bradley et al., 2011), Integrative Body-Mind Training (IBMT) (Tang, Tang, \& Posner, 2016). 270 However, as they seldomly been used in Chinese adolescents, their effectiveness in preventing 271 child abuse related SI should be further discussed.

272 The following limitations of our study should be noticed. At first, all our analytical results 273 were based on cross-sectional data, therefore, our major findings, especially the positive 
274 mediation of resilience in maltreatment-SI association are to be further corroborated by future

275 longitudinal studies with large sample sizes. Another limitation is that, as we only adjusted for

276 limited confounders when fitting the path model, residual confounding will exist inevitably.

277 Besides, childhood maltreatment was ascertained by self-reporting, so the possibility of recall

278 bias and information covering caused by stigma cannot be eliminated. Finally, we chose study

279 participants from a single city of Yunnan province, as they may not be representative to the 280 entire Chinese children and adolescent population, the extrapolation of study results should be 281 made with caution.

282 Despite all limitations stated above, our study is among the first attempts in investigating the 283 mediation of resilience in the association between childhood maltreatment and SI in Chinese 284 adolescents. The large sample size provides further consolidation to the validity of the results. 285 Our major findings suggest that building up psychological resilience, especially strengthening 286 emotion regulation skills and consolidating interpersonal support, might be useful in reducing the 287 risk of future suicide among Chinese adolescents who were childhood maltreatment victims. The 288 long-term effect of resilience in mediating the association between childhood maltreatment and 289 SI is to be corroborated by future prospective studies.

290

\section{Conclusions}

292 In conclusion, this population-based cross-sectional study revealed a strong association between 293 childhood maltreatment and SI, moreover, resilience played as a positive mediator in this 294 association. Among the five dimensions of resilience, emotion regulation and interpersonal 295 assistance presented the strongest mediation. Our findings suggested that resilience-oriented 296 strategies could be effective in reducing the risk of childhood maltreatment related suicidal 
297 behaviors among Chinese children and adolescents, especially intervention measures focusing on 298 strengthening emotion regulation skills and consolidating social support.

299

300 Acknowledgements

301 None.

302

303

304

305

306

307

308

309

310

\section{References}

Ahorsu DK, Adjaottor ES, Yeboah FA, Opoku Y. 2020. Mental health challenges in academia: comparison between students of the various educational levels in Ghana. Journal Of Mental Health 13: 1-8 DOI 10.1080/09638237.2020.1739253.

311

312

313

314

315

316

317

318

319

Beck AT, Steer RA, Ranieri WF. 1988. Scale for Suicide Ideation: psychometric properties of a self-report version. Journal of clinical psychology 44(4):499-505 DOI 10.1002/10974679(198807)44:4<499::aid-jclp2270440404>3.0.co;2-6.

Bernstein DP, Fink L, Handelsman L, Foote J, Lovejoy M, Wenzel K, Sapareto E, Ruggiero J. 1994. Initial reliability and validity of a new retrospective measure of child abuse and neglect. American Journal of Psychiatry 151(8):1132-1136 DOI 10.1176/ajp.151.8.1132.

Bradley B, Westen D, Mercer KB, Binder EB, Jovanovic T, Crain D, Wingo A, Heim C. 2011. Association between childhood maltreatment and adult emotional dysregulation 
320

321

322

323

324

325

326

327

328

329

330

331

332

333

334

335

336

337

338

339

340

341

in a low-income, urban, African American sample: Moderation by oxytocin receptor gene. Development and psychopathology 23(2):439-452 DOI $10.1017 / \mathrm{s} 0954579411000162$.

Centers for Disease Control and Prevention (CDC). 2017. Child Abuse and Neglect Prevention. from https://www.cdc.gov/ViolencePrevention/childmaltreatment/.(assessed 30 September 2017).

Chen JQ, Han P, Lian GL, Dunne, M P. 2010. Prevalence of childhood sexual abuse among 2508 college students in 6 provinces of China. Zhonghua liu xing bing xue za zhi 31(8):866-869. (In Chinese)

Cong EZ, Wu Y, Cai YY, Chen HY, Xu YF. 2019. Association of suicidal ideation with family environment and psychological resilience in adolescents. Zhongguo Dang Dai Er Ke Za Zhi 21(5):479-484. (In Chinese)

Cui S, Cheng Y, Xu Z, Chen D, Wang Y. 2010. Peer relationships and suicide ideation and attempts among Chinese adolescents. Child: Care, Health and Development 37(5):692702.

de Terte I, Stephens C. 2014. Psychological resilience of workers in high-risk occupations. Stress and Health 30 (5): 353-355

Dubowitz H, Thompson R, Proctor L, Metzger R, Black MM, English D, Poole G, Magder L. 2016. Adversity, Maltreatment, and Resilience in Young Children. Academic pediatrics 16(3):233-239 DOI 10.1016/j.acap.2015.12.005.

Eccles JS, Roeser RW. 2011. Schools as developmental contexts during adolescence. Journal of Research on Adolescence 21(1):225-241. 
342 Falgares G, Marchetti D, Manna G, Musso P, Oasi O, Kopala-Sibley DC, De Santis S,

343

344

345

346

347

348

349

350

351

352

353

354

355

356

357

358

359

360

361

362

363

364

Verrocchio MC. 2018. Childhood Maltreatment, Pathological Personality Dimensions, and Suicide Risk in Young Adults. Frontiers in psychology 9:806 DOI 10.3389/fpsyg.2018.00806.

Fergus S, Zimmerman MA. 2005. Adolescent resilience: A framework for understanding healthy development in the face of risk. Annual review of public health 26:399-419 DOI 10.1146/annurev.publhealth.26.021304.144357

Fry D, McCoy A, Swales D. 2012. The Consequences of Maltreatment on Children's Lives: A Systematic Review of Data From the East Asia and Pacific Region. Trauma Violence \& Abuse 13(4) DOI 209-233.10.1177/1524838012455873.

Gorrese A, Ruggieri R. 2012. Peer attachment: A meta-analytic review of gender and age differences and associations with parent attachment. Journal of Youth and Adolescence 41(5):650-672

Hong CH. 2017. The Moderating Effect of Ego-Resilience and the Mediating Effect of Social Support in the Relationship between Abuse Experience and Complex PTSD. The Korean Journal of Developmental Psychology 30(1):1-19

Hu Y, Gan Y. 2008. Development and Psychometric Validity of the Resilience Scale for Chinese Adolescents. Acta Psychologica Sinica (08):902-912. (In Chinese)

Ji K, Finkelhor D. 2015. A meta-analysis of child physical abuse prevalence in China. Child abuse \& neglect 43:61-72 DOI 10.1016/j.chiabu.2014.11.011.

Lee SW, Bae GY, Rim HD, Lee SJ, Chang SM, Kim BS, Won S. 2018. Mediating Effect of Resilience on the Association between Emotional Neglect and Depressive Symptoms. Psychiatry investigation 15(1):62-69.

Peer) reviewing PDF | (2021:01:57531:2:0:NEW 2 Jun 2021) 
365 Meng X, Fleury MJ, Xiang YT, Li M, D'Arcy C. 2018. Resilience and protective factors

366

367

368

369

370

371

372

373

374

375

376

377

378

379

380

381

382

383

384

385

among people with a history of child maltreatment: a systematic review. Social psychiatry and psychiatric epidemiology 53(5):453-475 DOI 10.1007/s00127-018-1485-2.

Miller AB, Esposito-Smythers C. 2013. How Do Cognitive Distortions and Substance-Related Problems Affect the Relationship Between Child Maltreatment and Adolescent Suicidal Ideation? Psychology of violence 3(4):340-353 DOI 10.1037/a0031355.

Miller AB, Jenness JL, Oppenheimer CW, Gottleib ALB, Young JF, Hankin BL. 2017. Childhood Emotional Maltreatment as a Robust Predictor of Suicidal Ideation: A 3-Year Multi-Wave, Prospective Investigation. Journal of abnormal child psychology 45(1):105116 DOI 10.1007/s10802-016-0150-Z.

Mishara BL. 1999. Conceptions of death and suicide in children ages 6-12 and their implications for suicide prevention. Suicide \& life-threatening behavior 29(2):105-118

Mizuta A, Suzuki A, Yamagata Z, Ojima T. 2017. Teachers' support and depression among Japanese adolescents: a multilevel analysis. Social Psychiatry and Psychiatric Epidemiology 52 (2):211-219.

Olsson CA, Bond L, Burns JM, Vella-Brodrick DA, Sawyer SM. 2003. Adolescent resilience: a concept analysis. Journal of Adolescence 26(1):1-11

Patel NK, Nivethitha L, Mooventhan A. 2018. Effect of a Yoga Based Meditation Technique on Emotional Regulation, Self-compassion and Mindfulness in College Students. Explore-the Journal of Science And Healing 14(6):443-447 DOI 10.1016/j.explore.2018.06.008.

Peer) reviewing PDF | (2021:01:57531:2:0:NEW 2 Jun 2021) 
386 Poole JC, Dobson KS, Pusch D. 2017. Childhood adversity and adult depression: The

387

388

389

390

391

392

393

394

395

396

397

398

399

400

401

402

403

404

405

406

407

408 protective role of psychological resilience. Child abuse \& neglect 64:89-100 DOI 10.1016/j.chiabu.2016.12.012.

Pu S, Setoyama S, Noda T. 2017. Association between cognitive deficits and suicidal ideation in patients with major depressive disorder. Scientific report 7(1):11637 DOI $10.1038 / \mathrm{s} 41598-017-12142-8$.

Rafiq S, Campodonico C, Varese F. 2018. The relationship between childhood adversities and dissociation in severe mental illness: a meta-analytic review. Acta psychiatrica Scandinavica 138(6):509-525 DOI 10.1111/acps.12969.

Rehan W, Antfolk J, Johansson A, Jern P, Santtila P. 2017. Experiences of severe childhood maltreatment, depression, anxiety and alcohol abuse among adults in Finland. PLoS One 12(5):e0177252 DOI 10.1371/journal.pone.0177252.

Salama E, Castaneda AE, Suvisaari J, Rask S, Laatikainen T, Niemela S. 2020. Substance use, affective symptoms, and suicidal ideation among Russian, Somali, and Kurdish migrants in Finland. Transcultural psychiatry DOI 10.1177/1363461520906028.

Shalev A, Porta G, Biernesser C, Zelazny J, Walker-Payne M, Melhem N, Brent D. 2019. Cortisol response to stress as a predictor for suicidal ideation in youth. Journal of Affective Disorders. 257:10-16 DOI 10.1016/j.jad.2019.06.053.

Stice E, Rohde P, Gau J, Ochner C. 2011. Relation of depression to perceived social support: Results from a randomized adolescent depression prevention trial. Behaviour Research and Therapy 49(5):361-366

Stickley A, Waldman K, Ueda M, Koyanagi A, Sumiyoshi T, Narita Z, Inoue Y, DeVylder JE, Oh H. 2020. Childhood neglect and suicidal behavior: Findings from the National 
409

410

\section{1}

412

413

414

415

Comorbidity Survey Replication. Child abuse \& neglect 103:104400 DOI 10.1016/j.chiabu.2020.104400.

Sveticic J, De Leo D. 2012. The hypothesis of a continuum in suicidality: a discussion on its validity and practical implications. Mental Illness 4(2):e15

Szanto K, Mulsant BH, Houck P, Dew MA, Reynolds CF. 2003. Occurrence and course of suicidality during short-term treatment of late-life depression. Archives of general psychiatry 60(6):610-617 DOI 10.1001/archpsyc.60.6.610.

Tan L, Xia T, Reece C. 2018. Social and individual risk factors for suicide ideation among Chinese children and adolescents: A multilevel analysis. International Journal of Psychology 53(2):117-125 DOI 10.1002/ijop.12273.

Tang YY, Tang R, Posner MI. 2016. Mindfulness meditation improves emotion regulation and reduces drug abuse. Drug and alcohol dependence 163:S13-S18 DOI 10.1016/j.drugalcdep.2015.11.041.

Wiklander M, Samuelsson M, Asberg M. 2003. Shame reactions after suicide attempt. Scandinavian journal of caring sciences 17(3):293-300 DOI 10.1046/j.14716712.2003.00227.x.

Wilkinson RB. 2004. The role of parental and peer attachment in the psychological health and self-esteem of adolescents. Journal of Youth and Adolescence 33:479-493.

WHO. 2018. LIVE LIFE: Preventing Suicide. https://www.who.int/publications/i/item/live-lifepreventing-suicide. (assessed 2 June 2021).

Xiao Y, Chen Y, Meng Q, Tian X, He L, Yu Z, Wang Y. 2019. Suicide ideation and suicide plan in Chinese left-behind children: Prevalence and associated factors. Journal of Affective Disorders 257:662-668 DOI 10.1016/j.jad.2019.07.072. 
432 Yearwood K, Vliegen N, Chau C, Corveleyn J, Luyten P. 2019. When do peers matter? The 433 moderating role of peer support in the relationship between environmental adversity, 434 complex trauma, and adolescent psychopathology in socially disadvantaged adolescents. $435 \quad$ Journal of adolescence 72:14-22

436 Zhang J, Brown GK. 2007. Psychometric Properties of the Scale for Suicide Ideation in China. 437 Archives of Suicide Research 11(2):203-210 DOI 10.1080/13811110600894652.

438 Zhao XF, Zhang YL, Li, L F, Zhou YF. 2005. Evaluation on reliability and validity of Chinese 439 version of childhood trauma questionnaire. Chinese Journal of Clinical Rehabilitation 440 16:209-211. (In Chinese) 
Table $\mathbf{1}$ (on next page)

Major characteristics of the study participants, Lincang, China, 2019. 
1 Table 1 Major characteristics of the study participants, Lincang, China, 2019.

\begin{tabular}{|c|c|c|c|}
\hline Features & SI & Non-SI & All \\
\hline Gender $(N, \%)$ : Girls & $697(63.89)$ & $1,012(49.25)$ & $1,709(54.32)$ \\
\hline Age (Mean (SE)) & $13.74(0.06)$ & $13.09(0.05)$ & $13.31(0.04)$ \\
\hline \multicolumn{4}{|l|}{ Ethnicity $(N, \%)$} \\
\hline Han & $728(66.73)$ & $1,384(67.35)$ & $2,112(67.13)$ \\
\hline Yi & $127(11.64)$ & $238(11.58)$ & $365(11.60)$ \\
\hline Dai & $82(7.52)$ & $128(6.23)$ & $210(6.68)$ \\
\hline Other & $154(14.12)$ & $305(14.84)$ & $459(14.59)$ \\
\hline \multicolumn{4}{|l|}{ Residence $(N, \%)$} \\
\hline Township & $491(45.00)$ & $1,026(49.93)$ & $1,517(48.22)$ \\
\hline Village & $600(55.00)$ & $1,029(50.07)$ & $1,629(51.78)$ \\
\hline \multicolumn{4}{|l|}{ Grade $(N, \%)$} \\
\hline Primary school & $300(27.50)$ & $832(40.49)$ & $1,132(35.98)$ \\
\hline Junior high school & $378(34.64)$ & $691(33.63)$ & $1,069(34.00)$ \\
\hline Senior high school & $413(37.86)$ & $532(25.89)$ & $945(30.04)$ \\
\hline \multicolumn{4}{|l|}{ Study style $(N, \%)$} \\
\hline Day students & $368(33.73)$ & $913(44.43)$ & $1,281(40.72)$ \\
\hline Boarding students & $723(66.27)$ & $1,142(55.57)$ & $1,865(59.28)$ \\
\hline Father's age (Mean (SE)) & $42.76(0.18)$ & $42.02(0.13)$ & $42.27(0.10)$ \\
\hline Mother's age (Mean (SE)) & $39.94(0.16)$ & $39.25(0.11)$ & $39.49(0.09)$ \\
\hline \multicolumn{4}{|l|}{ Father's education level $(N, \%)$} \\
\hline Elementary school and below & $343(31.44)$ & $542(26.37)$ & $885(28.13)$ \\
\hline Junior high school and above & $633(58.02)$ & $1,299(63.21)$ & $1,932(61.41)$ \\
\hline \multicolumn{4}{|l|}{ Mother's education level $(N, \%)$} \\
\hline Elementary school and below & $401(36.76)$ & $676(32.90)$ & $1,077(34.23)$ \\
\hline Junior high school and above & $617(56.55)$ & $1,199(58.35)$ & $1,816(57.72)$ \\
\hline \multicolumn{4}{|l|}{ Marital status of the parents $(N, \%)$} \\
\hline Married & $909(83.32)$ & $1,797(87.45)$ & $2,706(86.01)$ \\
\hline Divorced & $87(7.97)$ & $130(6.33)$ & $217(6.90)$ \\
\hline Re-married & $62(5.68)$ & $78(3.80)$ & $140(4.50)$ \\
\hline Widowed & $33(3.02)$ & $47(2.29)$ & $80(2.54)$ \\
\hline \multicolumn{4}{|l|}{ Family income $(N, \%)$} \\
\hline Stable & $981(89.92)$ & $1,926(93.72)$ & $2,907(92.40)$ \\
\hline Unstable & $108(9.90)$ & $128(6.23)$ & $236(7.50)$ \\
\hline \multicolumn{4}{|c|}{ Childhood maltreatment (CTQ sores, Mean (SE)) } \\
\hline Emotional abuse & $8.66(0.11)$ & $6.33(0.04)$ & $7.13(0.05)$ \\
\hline Physical abuse & $6.59(0.08)$ & $5.82(0.04)$ & $6.09(0.04)$ \\
\hline Sexual abuse & $5.54(0.05)$ & $5.22(0.02)$ & $5.33(0.02)$ \\
\hline Emotional neglect & $11.75(0.15)$ & $9.43(0.09)$ & $10.23(0.08)$ \\
\hline Physical neglect & $8.03(0.09)$ & $6.83(0.05)$ & $7.25(0.05)$ \\
\hline \multicolumn{4}{|l|}{ Resilience (RSCA scores, Mean (SE)) } \\
\hline Combined score & $84.29(0.46)$ & $98.96(0.34)$ & $93.87(0.30)$ \\
\hline Goal concentration (Dimension 1) & $15.48(0.14)$ & $18.12(0.11)$ & $17.21(0.09)$ \\
\hline Emotional regulation (Dimension 2) & $17.58(0.16)$ & $23.02(0.11)$ & $21.14(0.10)$ \\
\hline
\end{tabular}


Positive perception (Dimension 3)

Family support (Dimension 4)

Interpersonal assistance (Dimension 5)
$13.20(0.11) \quad 13.78(0.09)$

$19.67(0.11) \quad 21.36(0.08)$

$18.36(0.17) \quad 22.67(0.11)$
$13.58(0.07)$

$20.78(0.06)$

$21.18(0.10)$

2 CTQ: Childhood Trauma Questionnaire;SI: Suicidal ideation. RSCA: the combined score of Resilience Scale 3 for Chinese Adolescents.

4 
Table 2 (on next page)

Results of multivariate regression models for associated factors of SI 
Table 2 Results of multivariate regression models for associated factors of SI

\begin{tabular}{|c|c|c|c|}
\hline \multirow{2}{*}{ Covariates } & Multivariate 1 & Multivariate 2 & Multivariate 3 \\
\hline & OR $(95 \% \mathrm{CI})$ & OR $(95 \% \mathrm{CI})$ & OR $(95 \% \mathrm{CI})$ \\
\hline Age $(+1$ year $)$ & $1.12(1.04,1.20)$ & $1.12(1.04,1.21)$ & $1.11(1.02,1.21)$ \\
\hline Father's age ( +5 years $)$ & $1.00(0.85,1.19)$ & $1.04(0.87,1.25)$ & $1.02(0.85,1.23)$ \\
\hline Mother's age ( +5 years $)$ & $1.08(0.93,1.25)$ & $1.06(0.93,1.21)$ & $1.09(0.95,1.26)$ \\
\hline \multicolumn{4}{|l|}{ Gender (Ref: Boys) } \\
\hline Girls & $2.09(1.76,2.47)$ & $1.96(1.67,2.31)$ & $2.07(1.78,2.42)$ \\
\hline \multicolumn{4}{|l|}{ Study style (Ref: Day students) } \\
\hline Boarding students & $0.56(0.47,0.67)$ & $0.65(0.53,0.80)$ & $0.63(0.51,0.78)$ \\
\hline \multicolumn{4}{|c|}{ Father's education level (Ref: Elementary and below) } \\
\hline Junior high school and above & $0.96(0.79,1.16)$ & $1.07(0.90,1.27)$ & $1.10(0.93,1.29)$ \\
\hline \multicolumn{4}{|c|}{ Marital status of the parents (Ref: Married) } \\
\hline \multicolumn{4}{|l|}{ Resilience (Ref: RSCA scores < $<4$ ) } \\
\hline RSCA scores $\geq 94$ & & $0.17(0.14,0.22)$ & $0.20(0.16,0.26)$ \\
\hline
\end{tabular}

4 
RSCA: the combined score of Resilience Scale for Chinese Adolescents. 
Figure 1

The process for sampling and study participants selection

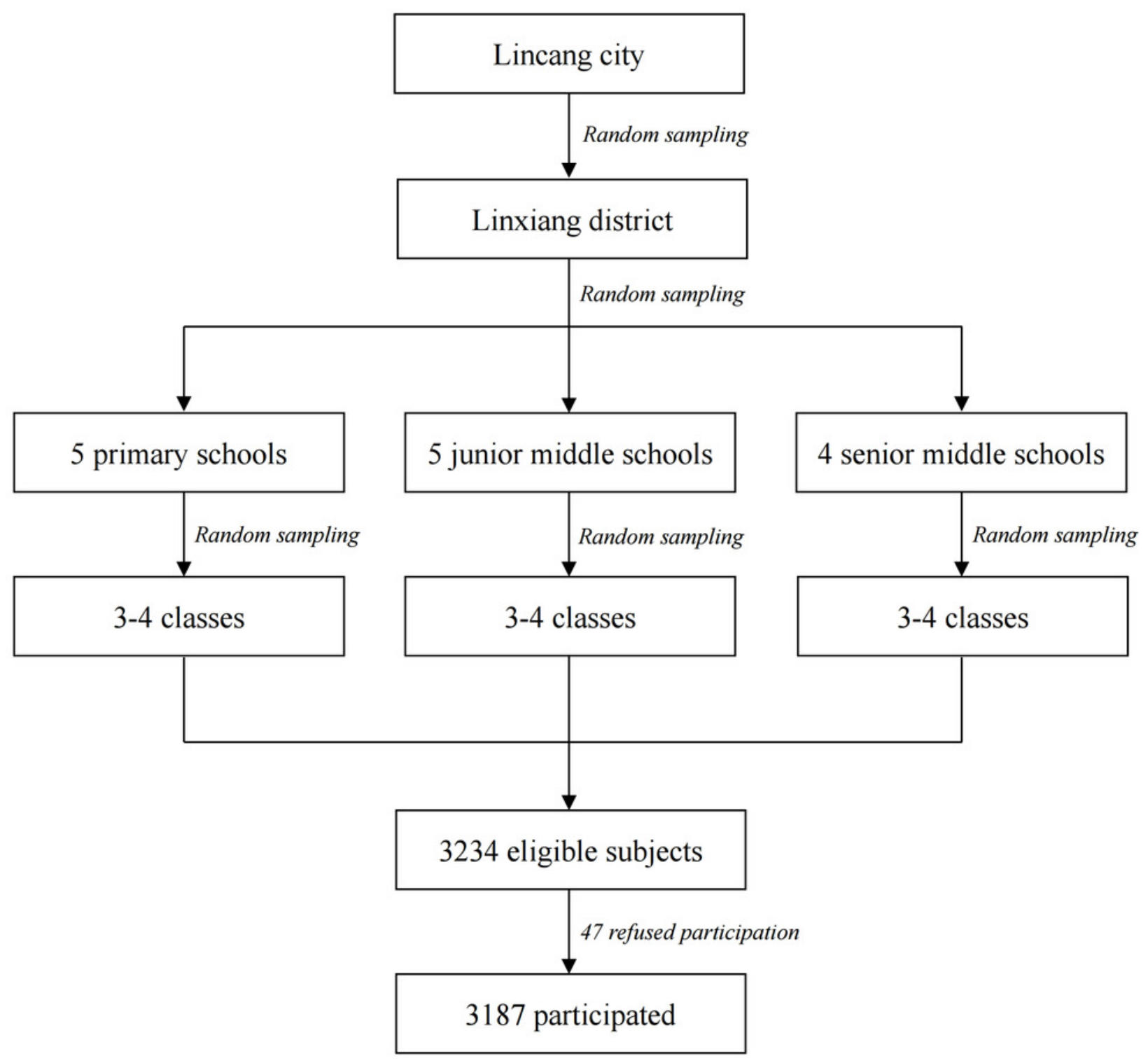


Figure 2

Adjusted ORs with $95 \% \mathrm{Cls}$ of SI (suicidal ideation) for types of childhood maltreatment. Adjusted for age, gender, study style, marital status of the parents, father's education level

OR $\quad 95 \% \mathrm{Cl}$

Emotional abuse (EA) $\quad 3.01 \quad$ (2.37 3.82)

Emotional neglect (EN) 1.78

Sexual abuse (SA) $\quad 1.68 \quad(1.21 \sim 2.33)$

Physical abuse (PA) $1.53 \quad(1.24 \sim 1.88)$

Physical neglect (PN) $1.34 \quad(1.14 \sim 1.56)$

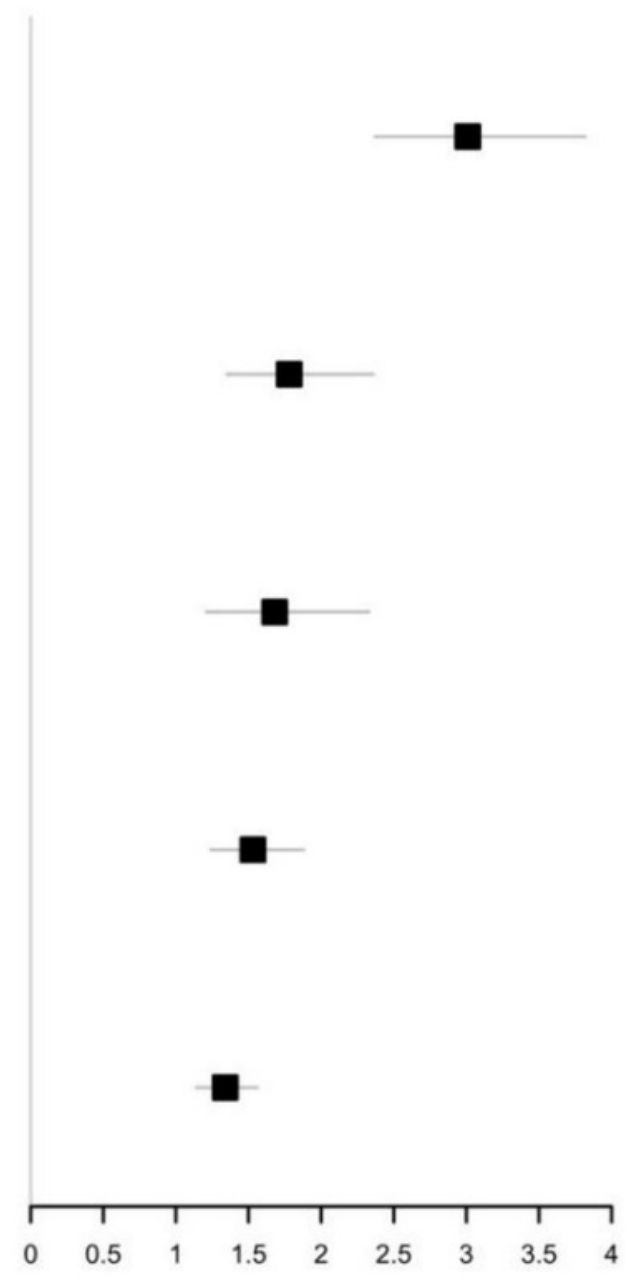


Figure 3

The hypothetical path model of 5 types of childhood maltreatment, resilience and SI

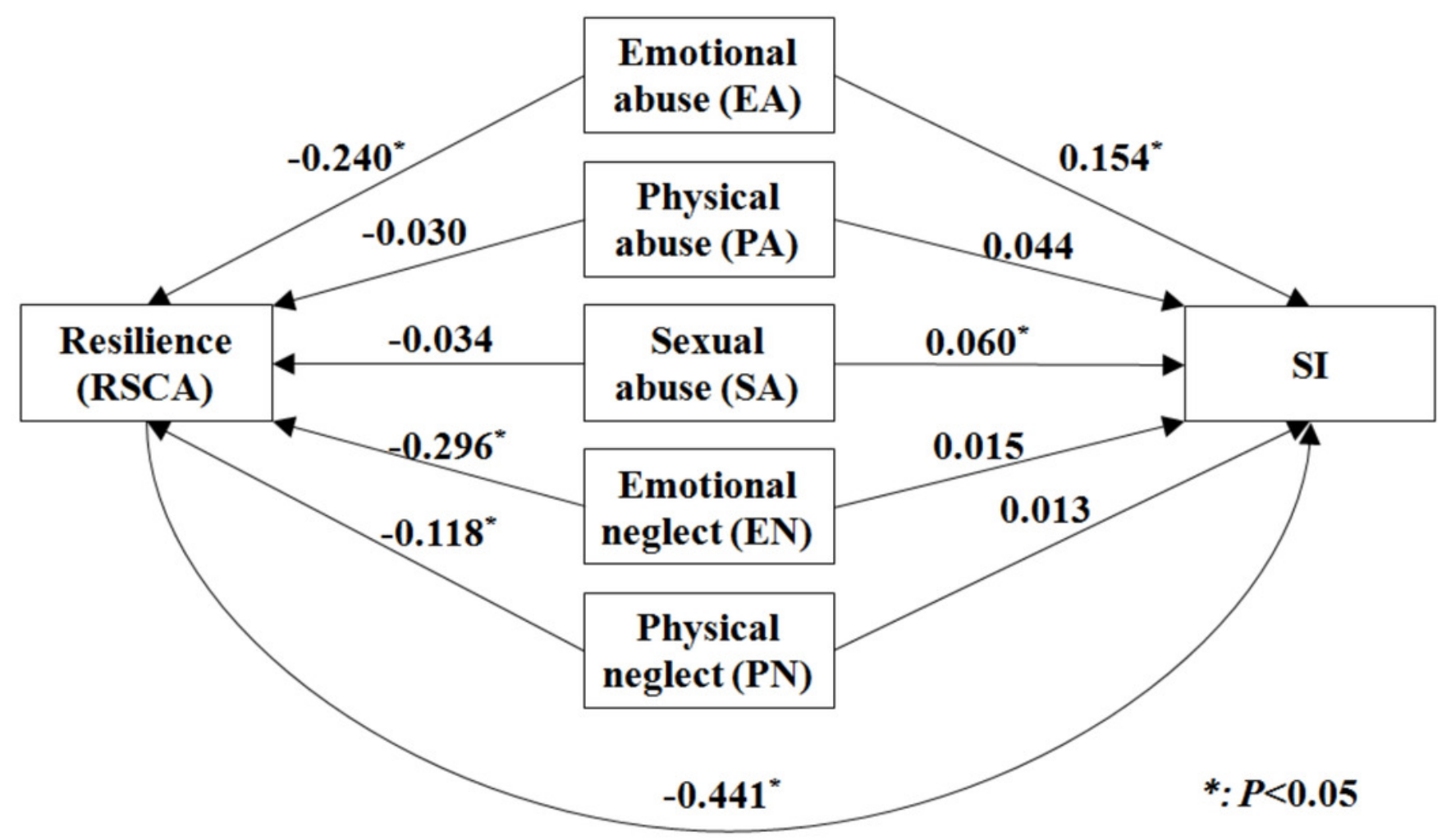


Figure 4

Proportion of mediating effect in association of EA, PA, PN and SI by different dimensions of resilience.

EA: Emotional abuse. PA: Physical abuse. PN: Physical neglect. 


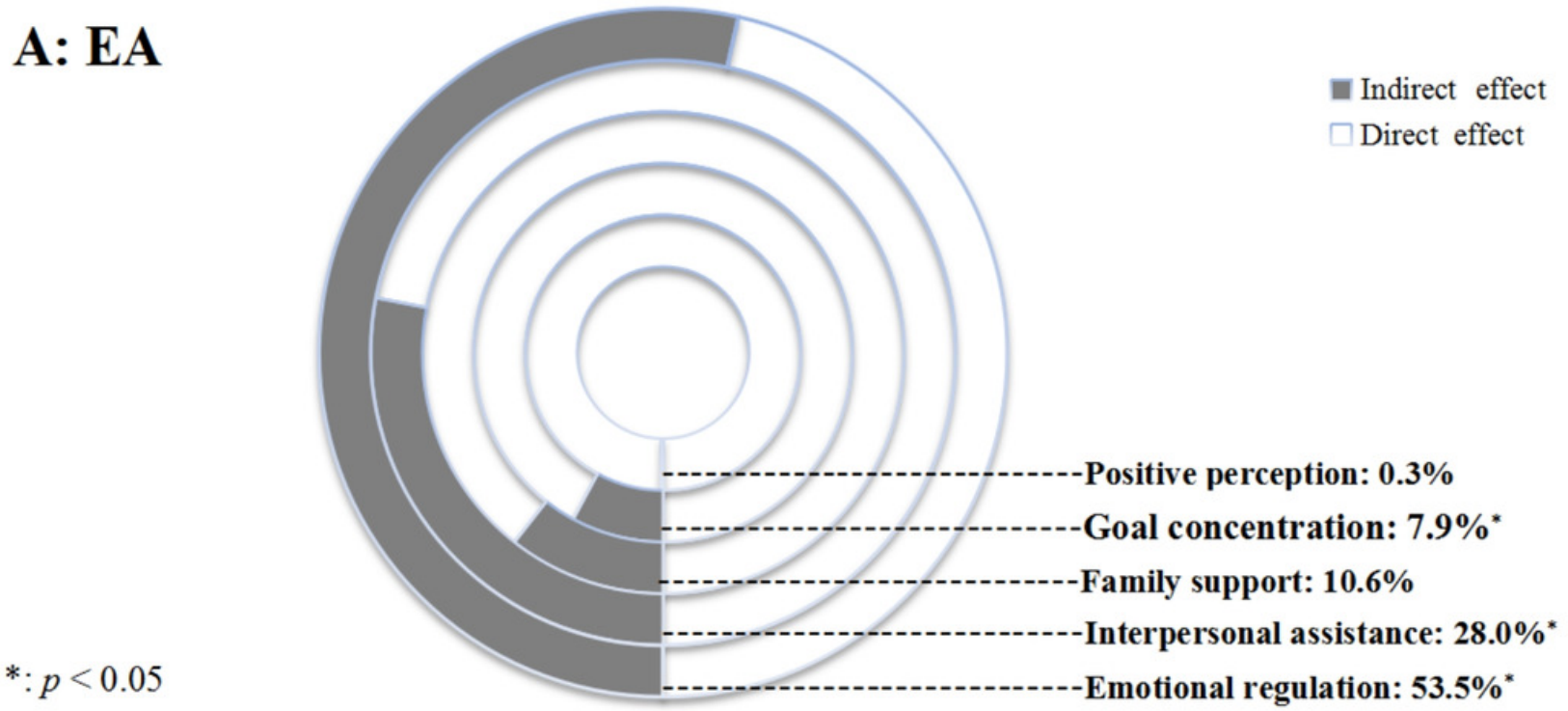

\section{B: EN}

$*: p<0.05$

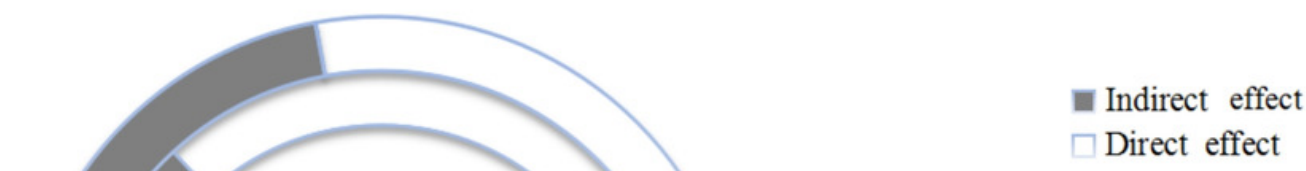

\section{C: PN}

$*: p<0.05$

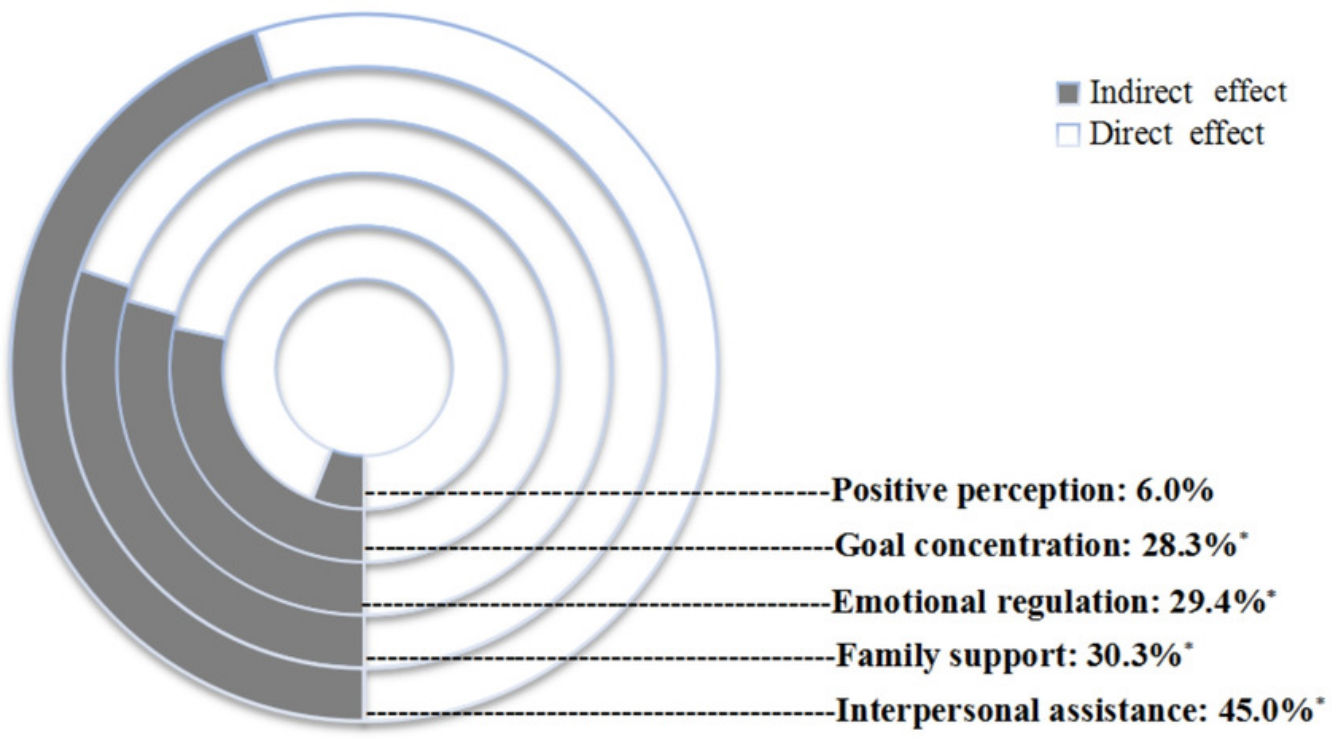

-Positive perception: $6.4 \%$

Family support: $\mathbf{3 1 . 3 \%}$ *

Goal concentration: $35.2 \%$ *

Emotional regulation: $38.2 \%{ }^{*}$

-Interpersonal assistance: $47.1 \%{ }^{*}$

Positive perception: $6.0 \%$

Goal concentration: $\mathbf{2 8 . 3 \%}$

Family support: $30.3 \%$ *

Interpersonal assistance: $\mathbf{4 5 . 0 \%}{ }^{*}$ 